\title{
Challenges in Smart Spaces: Aware of users, preferences, behaviours and habits
}

\author{
Pedro Oliveira ${ }^{1},{ }^{2}$, Paulo Novais ${ }^{1}$, and Paulo Matos ${ }^{2}$ \\ 1 Algoritmi Centre/University of Minho, Department of Informatics, Braga, Portugal, \\ 2 Institute Polytechnic of Bragança, Dep. of Informatics and Communications, \\ Bragança, Portugal
}

\begin{abstract}
There are new opportunities for research in the field of smart environments that should be explored. The concepts of smart homes and home automation, are currently in growing expansion in the scientific and research point of view, as the market demands for better solutions in this field. Users want that those spaces smartly adapt to their preferences in a transparent way. This paper describes the process of planning, reasoning and modeling of a Smart Environment, using emerging wearable devices on the market (smart watches, fitness trackers, etc.) and newer technologies like NFC, BLE and Wi-Fi Direct. Enabling the user to optimize the efficiency, comfort, and safety at the environments.
\end{abstract}

Keywords: AmI, smart-environments, wearables, wireless, multi-agent

\section{Introduction}

The aim of this work is to take advantage of emerging technologies available in the market that support the so-called wearable devices [1], and the non-invasive particularity of these to, in an autonomous way, adapt the environment to the comfort parameters of each user (e.g. thermal, acoustic, air quality, light, sun exposure) [2]. Provide comfort according to the preferences of each individual, is a challenge and an opportunity to create innovative solutions and new paradigms in the context of Intelligent Environments [3].

\section{Problem Statement}

The problem/challenge, need or, on the economic perspective, opportunity, which aims to overcome, can be defined in general terms as the difficulty in optimizing automatic, transparent and in a non-invasive way for the user, the comfort conditions, both at domestic and professional level or even in the use of public spaces. When it comes to comfort, we can also talk about security, productivity or other.

This challenge currently has as main difficulty, the mobility of people, the disparity of habits, schedules and every individual comfort preferences [4]. The same is aggravated when depending on physiological conditions, derived from 
a large number of factors (tiredness, mood, etc.), user preferences often suffer significant changes, that current systems can not measure. Contextualize user preferences, it is a process involving many variables and different dimensions, which makes this a problem of high complexity.

In addition to the physiological conditions mentioned above, there are two critical/essential dimensions, these are the space (user location) and time. In the case of the space can be as an example, the differences between the preferences of a personal, professional, recreational or other environment. Contextualize the user location is essential to optimize the conditions of comfort and contribute to the performance and effectiveness of the solution.

The time dimension is equally critical. Because the comfort preferences will change over the course of the day, as well as the week or even the year. For example, the comfort preferences may be different between daytime and nighttime, or between weekday and weekend/holidays. In this dimension, it is also important to assess the changes over the year, that will have the influence of the seasons, which naturally also change quite the user's comfort preferences. In this paradigm, which is intended to be the superlative of the comfort, there are at least these three dimensions: time, space and user comfort preferences.

The time and space dimensions are critical to contextualize the user's personal preferences, and provide the necessary information that will allow assess future preferences in a useful time.

Figure 1, shows the scenario of an environment where it intends to develop this work. Explaining this figure, it can be seen the user who through its different devices (smartphone, wearable, and other compatible) communicates with the system, and for that can be used different technologies (Wi-Fi Direct, Near Field Control, Bluetooth Low Energy). Next, the system performs communication with the Cloud, to validate the information. And then the system will perform the management of the different components in the environment (climatization systems, security systems, other smart systems).

For a better understanding of the overall scenario in the user's daily life, figure 2 contextualizes the temporal and space dimensions present in this problem and already mentioned above. We can see that different user locations, combined with time context, naturally results in an environment with different characteristics. This kind of global scenario should also be addressed in this project.

\section{Proposed Solution}

This section deals with the goals to be achieved, and detail the plan defined to solve the challenge described in section 2 .

The aim of this project is to create a solution that takes advantage of emerging technologies on the market that support wearable devices (e.g. smartwatches, smartphones, fitness trackers) and the non-invasive characteristic of these, for collecting data in an autonomous and transparent way and without any need of intervention by the user. Specifically this project aims to achieve the following goals: 


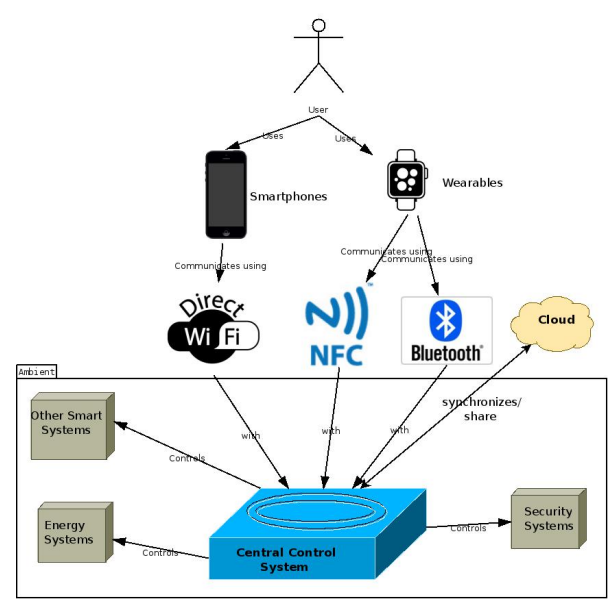

Fig. 1: Problem Statement

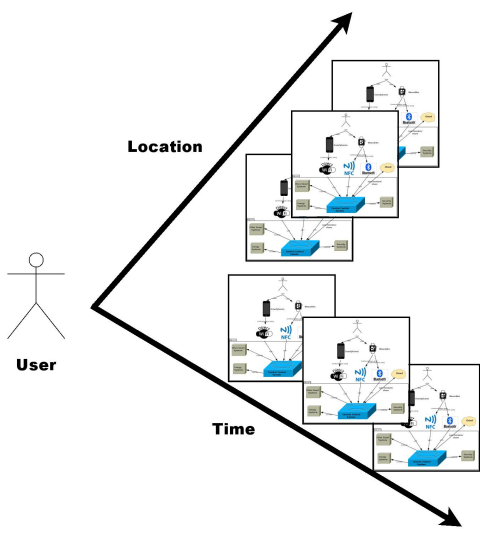

Fig. 2: Contextualization of Time/Environment Dimensions

- Characterize the different types of environments (Ambient Intelligence);

- Characterize the comfort in its different aspects and dimensions;

- Set a base architecture for a non-invasive system that takes advantage of emerging technologies and wearable devices data collection (smartwatches, smartphones, fitness trackers) for the intended purpose;

- Use of intelligent agents [5], to represent the different stakeholders, contexts and dimensions of the problem, who cooperate to achieve the optimal solution;

- Develop solutions that allow ubiquity in the identification of users and their comfort preferences, in an automatic and transparent way, enhancing the integration between space, time and user;

- Define an architecture solution to facilitate the user's interaction with existing systems;

- Use an intelligent multi-agent system, to manage the possible conflicts of interest, namely between users of the same space;

- Apply the proposed prototype at a health facility and/or a higher education institution, taking advantage of the existing company's partnerships;

- Evaluate the prototype using real/simulated problems of conflict management, between different comfort preferences that distinct users that share the same space.

The process and learning model proposed to the system, is intended to be scientifically innovative, taking advantage of the latest research in this field and combining multiple factors and technologies described below:

- Use dynamically scaled priorities rules, which must have the information, considered essential for the correct functioning of the system, including the limits for the different parameters, like system reliability or user safety. 
- Use of multi-agent systems representing the different entities involved in the negotiation process, allowing an efficient outcome in different situations.

- Use of sensors collected information, and using machine learning techniques, including Sequence Discovery, Fuzzy Logic, Genetic Programming, Multi-Layer Perceptron, as described in [6], get information about user's habits in the environment.

- Context awareness, as described above since the context is entirely relevant in such systems.

- Use of logical sensors, there are three types of sensors used to assess the context in such systems: physical, virtual and logical.

That said, the practical applicability of this work, should result in the complete specification of an intelligent environment. To optimize the predictions of the solution proposed, an architecture for a multi-agent system was also defined.

\section{Preliminary Results and Future Work}

This project combines the use of the latest wireless communication technology with emerging wearable devices, and therefore optimize the everyday people lives and the industrial production environments. Is defined as a truly innovative project and fully applicable to industrial and domestic level. This work resulted in the complete specification of an architecture that supports the proposed solution, to solve the presented problem. It will now be implemented, tested and validated using real case studies, so as to gather statistical information to assess its effectiveness and performance in the context of application.

Acknowledgements: This work has been supported by COMPETE: POCI01-0145-FEDER-007043 and FCT Fundao para a Cincia e Tecnologia within the Project Scope: UID/CEC/00319/2013.

\section{References}

1. E. Fleisch, "What is the internet of things? an economic perspective," Economics, Management, and Financial Markets, no. 2, pp. 125-157, 2010.

2. E. H. Aarts and B. E. de Ruyter, "New research perspectives on ambient intelligence." JAISE, vol. 1, no. 1, pp. 5-14, 2009.

3. J. C. Augusto, V. Callaghan, D. Cook, A. Kameas, and I. Satoh, "Intelligent environments: a manifesto," Human-Centric Computing and Information Sciences, vol. 3 , no. 1 , pp. 1-18, 2013.

4. E. Aarts and F. Grotenhuis, "Ambient intelligence 2.0: Towards synergetic prosperity," Journal of Ambient Intelligence and Smart Environments, vol. 3, no. 1, pp. 3-11, 2011.

5. F. Bellifemine, A. Poggi, and G. Rimassa, "Developing multi-agent systems with jade," in Intelligent Agents VII Agent Theories Architectures and Languages. Springer, 2001, pp. 89-103.

6. K. I.-K. Wang, W. H. Abdulla, and Z. Salcic, "Ambient intelligence platform using multi-agent system and mobile ubiquitous hardware," Pervasive and Mobile Computing, vol. 5, no. 5, pp. 558-573, 2009. 